\title{
Decreased levels of vaspin and its potential association with cardiometabolic risk in patients with psoriasis: preliminary results
}

\author{
Paulina Kiluk¹, Anna Baran¹, Tomasz W. Kaminski², Magdalena Maciaszek³ ${ }^{3}$ Iwona Flisiak ${ }^{1}$
}

${ }^{1}$ Department of Dermatology and Venereology, Medical University of Bialystok, Bialystok, Poland 2Pittsburgh Heart, Lung and Blood Vascular Medicine Institute, University of Pittsburgh, Pittsburgh, USA ${ }^{3}$ Department of Infectious Diseases and Hepatology, Medical University of Bialystok, Bialystok, Poland

Adv Dermatol Allergol 2022; XXXIX (2): 307-315

DOI: https://doi.org/10.5114/ada.2021.103383

\begin{abstract}
Introduction: Omentin and vaspin are considered to have beneficial effects preventing the development of metabolic disorders which are common comorbidities in psoriasis.

Aim: To evaluate the serum level of these adipokines in psoriatic patients and elucidate possible associations with disease activity, metabolic or inflammatory parameters and systemic treatment.

Material and methods: Thirty-three patients with active plaque-type psoriasis and 11 healthy controls participated in the study. Blood samples were collected before and after 3 months of treatment with acitretin or methotrexate. Results: Serum vaspin concentration in psoriatic patients was significantly lower than in the control group $(p<0.05)$. No correlation between adipokines and severity of disease evaluated with PASI was found. However, median vaspin levels decreased with the severity of skin lesions and the omentin level was higher in patients with severe disease versus those with moderate form $(p<0.05)$. The vaspin level correlated with BMI of psoriatic patients $(p<0.05)$, with cholesterol and triglycerides levels ( $p=0.054, p=0.049$, respectively). No significant effect of systemic treatment on omentin levels was found. Regarding vaspin, we observed an upward trend in its concentration after treatment. Conclusions: Omentin and vaspin may play a modulating role in the systemic inflammation present in psoriasis and thus may contribute to the development of metabolic complications.
\end{abstract}

Key words: psoriasis, vaspin, omentin, cardiometabolic diseases, obesity.

\section{Introduction}

Psoriasis is a chronic inflammatory skin disease, characterized by increased proliferation of keratinocytes, disturbed epidermal cell differentiation and increased angiogenesis. Psoriasis is considered not only as a disease affecting the skin and joints but as a systemic inflammatory disease. Metabolically driven inflammation present in psoriasis is associated with a higher incidence of cardiometabolic disorders (CMDs) such as diabetes mellitus (DM), hypercholesterolemia, as well as cardiovascular diseases (CVD) [1]. Particular attention in this group of patients should be paid to the risk of developing potentially fatal CVD such as myocardial infarction or thromboembolic events. It was proven that the accelerated development of cardiovascular complications in patients with psoriasis leads to a 5-year reduction in their life expectancy [2]. Genetic background, insulin resistance (IR), dyslipidemia, oxidative stress, angiogenesis, secretion of adipokines are mentioned as possible causes of coexistence of CMDs and psoriasis [3]. Nowadays, more and more attention has been paid to the role played by adipose tissue in the pathogenesis of systemic inflammation. Adipose tissue affects internal organs, systemic metabolic pathways and the cardiovascular system through secretion of adipokines (such as for example leptin, adiponectin, visfatin, resistin, omentin, vaspin) and cytokines (tumor necrosis factor- $\alpha$, interleukin-6) [4]. The growing problem of obesity is also visible among patients with psoriasis [5]. It is presumed that adipokines secreted by adipose tissue can be involved in chronic inflammation leading to chronic skin lesions and the development of metabolic complications [6, 7]. However, newer adipokines are being constantly explored. Relatively new ones that show a protective effect against metabolic disorders are omentin and vaspin. Vaspin (VASP, SERPIN 12) is a recently discovered adipokine that

Address for correspondence: Paulina Kiluk MD, Department of Dermatology and Venereology, Medical University of Bialystok, Bialystok, Poland, e-mail: paulina.kiluk@umb.edu.pl Received: 10.12.2020, accepted: 7.01.2021. 
has been isolated from the visceral adipose tissue of OLETF (Otsuka Long-Evans Tokushima Fatty) rats, an animal model of abdominal obesity associated with type 2 diabetes (T2D). VASP is a serine protease inhibitor consisting of 375 amino acids [8]. The exact mechanism of its action has not been determined, but it was reported that a likely target for vaspin is the kallikrein-related peptidase 7 (hK7) [9]. In experimental studies, improvement in glucose tolerance and insulin sensitivity was observed after administration of recombinant vaspin in DIO (dietinduced-obesity) mice. It is supposed that vaspin acts as an insulin sensitizer with anti-inflammatory effects and might display a compensatory mechanism in response to decreased insulin sensitivity $[8,10]$. As obesity is one of the main factors contributing to the development of CMDs also in psoriasis, the possible protective effect of vaspin in their manifestation is still debatable. The data concerning vaspin impact on psoriasis pathogenesis are available, but still not fully clear and unambiguous.

Omentin was isolated from the visceral adipose tissue by Yang et al. [11]. It includes two isoforms, the first of which (omentin-1) is the main circulating isoform and contains 313 amino acids. The adipokine is mainly expressed in visceral adipose tissue, predominantly in stromal-vascular cells (SVC) rather than subcutaneous adipose tissues in humans $[12,13]$. The gene for omentin is located in the 1q22-a23 chromosomal region which has been previously linked to T2D in several populations. These data suggest that omentin might be a candidate gene for T2D susceptibility in humans [12]. Moreover, it is reported that omentin increases insulin-mediated glucose uptake by activating the protein kinase Akt or protein kinase B [13]. It is also suspected that the adipokine exerts anti-inflammatory effects in endothelial cells. As a consequence of the activation of the eNOS/NO path, vasodilation occurs in isolated blood vessels [14, 15]. Therefore, omentin might be involved in obesity and its metabolic consequences including cardio-vascular complications, which are common comorbidities in psoriasis.

\section{Aim}

Due to the increasing prevalence of obesity and cardiometabolic complications in psoriatic patients, the aim of our study was to assess the serum levels of discussed adipokines in patients with psoriasis and evaluate their relationship with disease severity, metabolic and inflammatory parameters as well as potential association with CMDs and influence of systemic treatment.

\section{Material and methods}

To our prospective research we enrolled 33 patients (21 males and 12 females) with a flare of plaque-type psoriasis, and 11 sex-, age- and body mass index (BMI)matched healthy controls. Patients with other types of psoriasis, chronic metabolic or inflammatory diseases, malignancy, and pregnancy were excluded. Psoriasis area and severity index (PASI) has been estimated by the same investigator in all patients. The study group was divided depending on the PASI severity into 3 subgroups: mild (PASI 1) score under 10 points, moderate (PASI 2) between 10 and 20 points and severe (PASI 3) above 20 points. Body mass index (BMI) was evaluated as weight/ height ${ }^{2}\left(\mathrm{~kg} / \mathrm{m}^{2}\right)$. We divided patients for groups according to BMI, group 0 means the controls, BMI 1 includes 10 normal-weight $\left(18.5-24.9 \mathrm{~kg} / \mathrm{m}^{2}\right)$ psoriatics, BMI 2 indicates overweight (BMI $25.0-29.9 \mathrm{~kg} / \mathrm{m}^{2}$ ) and consists of 9 patients, BMI 3 - obesity $\left(\mathrm{BMI}>30 \mathrm{~kg} / \mathrm{m}^{2}\right)$ noted in 14 subjects from the study group. Blood samples have been collected before starting systemic treatment and repeated after therapy. Treatment was conducted with methotrexate (MTX) 15 mg/week (19 patients) or acitretin (14 persons) in a bodyweight dose of $0.5 \mathrm{mg} / \mathrm{kg} /$ day. The therapy lasted 3 months. All participants gave their signed informed consent before participation. The study was approved by the Bioethical Committee of the Medical University of Bialystok (Protocol number R-I-002/354/2015) and was in accordance with the principle of the Helsinki Declaration.

\section{Serum collection}

Fasting blood samples were obtained from the study and control group, using vacutainer tubes and allowed to clot for $30 \mathrm{~min}$. Samples were centrifuged for $15 \mathrm{~min}$ at $2000 \mathrm{~g}$, and then separated serum was frozen immediately and preserved at $-80^{\circ} \mathrm{C}$ until analysis. Blood samples for biochemical tests and blood counts were collected at the same time to other tubes and were performed by routine laboratory techniques using an automated analyzer. Serum levels of assay parameters were measured using validated and calibrated Bio-Plex 200 System provided by Bio-Rad. In our research we used BioPlex Pro RBM Human Metabolic Panel 2 which includes 8 magnetic bead-based assays to measure among other omentin and vaspin.

\section{Statistical analysis}

The normality of obtained distribution was tested using the Shapiro-Wilk test and quantitative data were expressed as mean \pm SD. The non-Gaussian data were presented as a median (full range). The Student's $t$ test or nonparametric Mann-Whitney test were used to compare differences between the psoriasis group and the control group, whereas for binary data $\chi^{2}$ test was used. The analysis of variance (ANOVA) or Kruskal-Wallis test was used to check differences between the psoriasis subgroups and followed by Bonferroni post hoc analysis when appropriate. The correlations between studied variables were determined by Spearman's rank correlation analysis. A two-tailed $p$ value $<0.05$ was considered to be 
statistically significant. Computations were performed using GraphPad 8 Prism Software (GraphPad Software, (A, USA). The power of the analysis was estimated using StatMate 2 Software (GraphPad Software, CA, USA).

\section{Results}

The study included 33 patients with active plaquetype psoriasis, 12 women and 21 men, with the mean age of 54 (24-85) and 11 healthy volunteers matched for age, weight, and BMI. Median BMI of psoriatic patients was $28.86 \mathrm{~kg} / \mathrm{m}^{2}(17.3-43)$. Severity of psoriasis expressed by median PASI score was 15 (5.4-32.7) before treatment and 3.4 (0.7-15) after therapy. Baseline characteristics of the control group and patients are summarized in Table 1. The median vaspin serum concentration in psoriatic patients before treatment was significantly $(p<0.05)$ lower than in the control group (Figure 1, Table 2). The serum level of omentin in the study group before treatment was insignificantly higher than in controls, however with an upward trend (Table 2). There was no correlation between the studied adipokines and gender in the study. However, a significant positive correlation between the serum omentin concentration and age was found in the group of psoriasis patients $(p=0.003)$. We found no statistically significant correlation between the vaspin level before treatment and severity of psoriasis expressed by PASI $(p<0.05)$ (Tables 3 and 4$)$. Interest-
Table 1. Baseline characteristics of the control group and patients

\begin{tabular}{lcc}
\hline Parameter & Controls & Patients \\
\hline Age [years] & $54.36 \pm 9.11$ & $54.15 \pm 16.77$ \\
\hline Height $[\mathrm{cm}]$ & $165.9 \pm 8.83$ & $171.5 \pm 10.01$ \\
\hline Weight $[\mathrm{kg}]$ & $69.36 \pm 15.19$ & $84.8 \pm 20.93(p=0.0646) \mathrm{NS}$ \\
\hline BMI $\left[\mathrm{kg} / \mathrm{m}^{2}\right]$ & $24.95 \pm 3.59$ & $28.86 \pm 6.41(p=0.0627) \mathrm{NS}$ \\
\hline
\end{tabular}

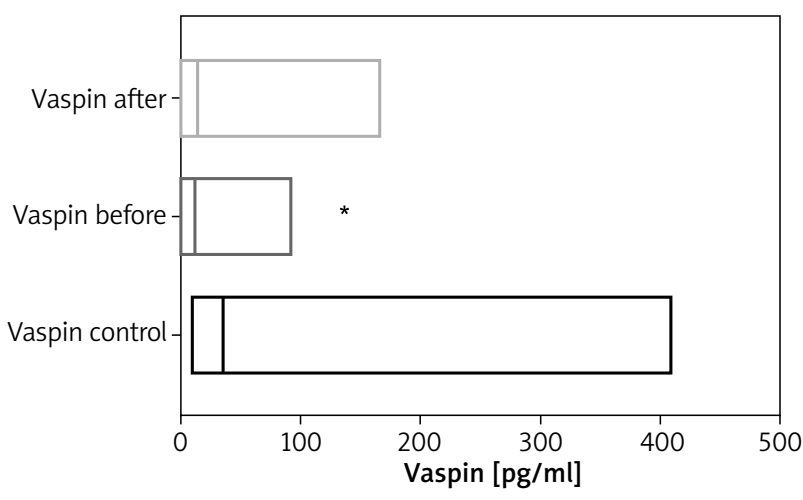

*Means a statistical significance between controls and given patients group.

Figure 1. Comparison of the levels of vaspin between controls and patients before and after treatment (point 1741 has not been included in graphics, it is beyond the scale)

Table 2. Comparison of levels of omentin and vaspin between controls and patients before and after treatment. Concentrations of omentin, vaspin in groups treated with acitretin or methotrexate

\begin{tabular}{lcccc}
\hline Parameter & Controls & Acitretin & Methotrexate & Patients \\
\hline Omentin $[\mathrm{ng} / \mathrm{ml}]:$ & & & \\
\hline Before treatment & $7.22(3.59-39.89)$ & $8.27(0.04-22.36)$ & $12.80(0.54-69.13)$ & $11.27(0.54-69.13)$ \\
\hline After treatment & - & $10.2(0.02-21.81)$ & $10.3(3.06-75.01)$ & $10.39(2.25-75.01)$ \\
\hline Vaspin $[\mathrm{pg} / \mathrm{ml}]:$ & & & \\
\hline Before treatment & $35.21(7.88-411.60)$ & $6.46(0.01-15.36)^{\star \star \star}(<0.0001)^{\# \# \#}$ & $28.73(6.85-94.14)$ & $11.53(0.01-94.14)^{\star}(0.042)$ \\
\hline After treatment & - & $5.91(0.38-28.42)^{\star \star \star}(<0.0001)^{\# \# \#}$ & $27.79(1.40-1746.00)$ & $15.08(0.38-1746.00)(0.061)$
\end{tabular}

Italic shows the existence of a strong trend at the border of significance (due to small " $n$ " numbers). * ${ }^{* * * * *}$ means a statistical significance between controls and given patients group. ${ }^{\# \# A N O V A}$ analysis within vaspin subgroups.

Table 3. Presence of crucial correlations between baseline parameters and omentin, vaspin before and after treatment. Italic shows statistical significances between group Before treatment and After treatment

\begin{tabular}{lcccc}
\hline Parameter & \multicolumn{2}{c}{ Before treatment } & After treatmen \\
\cline { 2 - 5 } & Omentin & Vaspin & Omentin & -0.195 \\
\hline PASI Score & 0.028 & -0.122 & -0.244 & -0.098 \\
\hline Red blood cells & -0.126 & 0.130 & $-0.286(0.088)$ & -0.033 \\
\hline White blood cells & -0.177 & $0.435(0.008)$ & -0.003 & -0.114 \\
\hline C-reactive protein & -0.008 & 0.049 & $-0.348(0.047)$ & -0.146 \\
\hline Cholesterol total & -0.230 & $0.485(0.004)$ & -0.116 & $0.342(0.049)$ \\
\hline Triglycerides & -0.227 & &
\end{tabular}


Table 4. Concentrations of omentin, vaspin in groups based on PASI scoring regarding the examination at admission to the clinic and after treatment

\begin{tabular}{lccccc}
\hline Parameter & Protein/group & Controls $(n=11)$ & PASI $1(n=6)$ & PASI $2(n=14)$ & PASI $3(n=13)$ \\
\hline $\begin{array}{l}\text { Before } \\
\text { treatment }\end{array}$ & Omentin $[\mathrm{ng} / \mathrm{ml}]$ & $7.22(3.58-39.89)$ & $9.47(4.21-21.46)$ & $6.44(0.04-69.13)$ & $12.80(5.47-36.51) \wedge(0.048)$ \\
\cline { 2 - 6 } & Vaspin $[\mathrm{pg} / \mathrm{ml}]$ & $35.21(7.88-411.60)$ & $20.83(6.36-94.14)$ & $13.39(0.01-67.31)$ & $10.63(4.05-84.78)$ \\
\hline \multirow{2}{*}{$\begin{array}{l}\text { After } \\
\text { treatment }\end{array}$} & Omentin $[\mathrm{ng} / \mathrm{ml}]$ & - & $10.51(5.14-21.81)$ & $8.00(0.02-75.01)$ & $11.71(5.75-40.10)$ \\
\cline { 2 - 6 } & Vaspin $[\mathrm{pg} / \mathrm{ml}]$ & - & $10.84(1.4-77.98)$ & $8.55(0.38-1746.00)$ & $15.79(1.98-169.20)$ \\
\hline
\end{tabular}

^A significant difference between PASI 2 and PASI 3 subgroups in omentin concentrations. Italic shows the existence of a strong trend at the border of significance (due to small " $n$ " numbers).

Table 5. Concentrations of omentin, vaspin in groups based on body mass index (BMI) values regarding the examination at admission to the clinic and after treatment

\begin{tabular}{lccccc}
\hline Parameter & Protein/group & Controls $(n=11)$ & BMI I $(n=10)$ & BMI II $(n=9)$ & BMI III $(n=14)$ \\
\hline $\begin{array}{l}\text { Before } \\
\text { treatment }\end{array}$ & Omentin $[\mathrm{ng} / \mathrm{ml}]$ & $7.22(3.59-39.89)$ & $12.70(0.54-69.13)$ & $6.99(423.1-36.06)$ & $11.10(3.88-36.51)$ \\
\cline { 2 - 6 } & Vaspin $[\mathrm{pg} / \mathrm{ml}]$ & $35.21(7.88-411.6)$ & $\mathbf{9 . 8 1}\left(\mathbf{1 . 8 3 - 5 1 . 6 2 )}(\mathbf{0 . 0 6 2})^{\#}\right.$ & $6.6(0.01-28.73)^{* *}(0.0022)^{\#}$ & $\mathbf{3 0 . 1 4}(4.05-94.14)^{\#}$ \\
\hline \multirow{2}{*}{$\begin{array}{l}\text { After } \\
\text { treatment }\end{array}$} & Omentin $[\mathrm{ng} / \mathrm{ml}]$ & - & $9.08(3.06-75.01)$ & $8.32(0.02-44.39)$ & $11.744(5.75-40.10)$ \\
\cline { 2 - 6 } & Vaspin $[\mathrm{pg} / \mathrm{ml}]$ & - & $\mathbf{5 . 4 2}(1.40-1746.00)^{* \#}$ & $15.08(0.38-28.42)^{\#}$ & $32.83(1.98-169.20)^{\#}$ \\
\hline
\end{tabular}

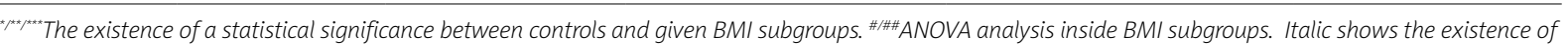
a strong trend at the border of significance (due to small " $n$ " numbers).

ingly, pre-treatment median vaspin levels decreased with the severity of skin lesions and the lowest was noticed in patients with severe psoriasis (Table 4). Serum omentin concentrations did not correlate with total PASI score but they were statistically significantly higher in patients with severe disease compared to those with moderate form ( $p<0.05)$ (Tables 3 and 4$)$. We noticed a statistically significant positive correlation between pre- and post-treatment vaspin levels and BMI of the patients ( $p<0.05 ; p=0.046, p=0.039$ respectively). In addition, there was a significant difference in vaspin concentrations between the control group and psoriatic overweight patients $(p<0.01)$. The study demonstrated statistically significant differences in vaspin levels between individual $\mathrm{BMI}$ subgroups and the control group in the Anova test. The lowest medium vaspin concentration was observed in overweight patients (BMI 2 subgroup), with the highest among obese psoriatics (BMI 3 subgroup) (Table 5). In the case of omentin, we did not find any significant correlations with patients total BMI or any trend in its levels between three BMI sub-groups (Table 5). After 3 months of systemic treatment, not only improvement of skin lesions in all studied patients was observed but also attenuation of inflammation expressed as a decrease in serum concentrations of inflammatory parameters (white blood count, C-reactive protein).

After systemic therapy of all patients, we observed an increasing trend in vaspin concentration, however without statistical significance $(p=0.061)$ (Table 2). Omentin levels before and after 3 months' therapy remained unchanged ( $p>0.05$ ) (Table 2). After treatment, the study revealed persistence of strong differences in vaspin concentrations between the control group and all three BMI subgroups expressed by the Anova test. Moreover, we noticed a significant difference in vaspin concentrations between the control group and psoriatics with normal body weight after treatment $(p<0.05)$ (Table 5). No significant relationship was found between adipokine levels in the control group and PASI subgroups (Table 4). Assessing the effect of treatment on vaspin or omentin levels in BMI and PASI subgroups, we did not find any statistically significant differences in adipokine levels in individual subgroups. However, we noticed that levels of vaspin decreased after treatment among PASI 1 and PASI 2 groups, contrary to a post-treatment vaspin level increase among PASI 3 group (Table 4). Moreover, we showed a decrease in the vaspin level after therapy among BMI 1 group, but its increase among BMI 2 and 3 groups. After division of patients into subgroups based on treatment strategy, we found no significant changes in vaspin and omentin levels before and after treatment in both acitretin and methotrexate groups ( $p>0.05)$ (Table 2). However, we noticed a statistically significant difference in vaspin levels between the control and acitretin groups before treatment $(p<0.01)$, this relationship was maintained even after treatment $(p<0.01)$ (Table 2).

We have also correlated serum of vaspin and omentin with biochemical, inflammatory and morphological parameters. Among all the correlations made, it is worth noting positive significant correlations between the serum vaspin level and total cholesterol $(R=0.485$, $p=0.004)$, triglycerides (TG) $(R=0.342, p=0.049)$ and white blood cells (WBC) $(R=0.435, p=0.008)$ in patients with psoriasis. This correlation was not present after treatment. 


\section{Discussion}

Adipokines derived from adipose tissue affect many tissues and organs including the brain, liver, cardiovascular system, skeletal muscle and others [16, 17]. They participate in multiple metabolic processes such as appetite regulation, maintaining energy balance, regulation of fat and carbohydrate metabolism, insulin sensitivity and insulin secretion, vascular remodeling and inflammation [18-20]. Excess visceral adipose tissue through secreted cytokines and adipokines contributes to the development of insulin resistance, DM, non-alcoholic fatty liver disease (NAFLD), atherosclerosis and hypertension, thus increasing the risk of developing cardiovascular diseases [16, 17]. Psoriasis, just as obesity, is characterized by the presence of the low-grade chronic inflammation state, which increases the risk of developing metabolic disorders. Therefore, adipokines in these two states play a modulatory or even a shared role. The relationship between obesity and psoriasis represent a challenging issue. It is still debatable whether obesity is entirely a consequence of psoriasis or it may be an independent risk factor for the development of this condition [21]. Omentin and vaspin belong to anti-inflammatory adipokines whose participation in psoriasis has been the subject of single, inconsistent studies.

It is suggested that vaspin is the metabolic regulator of fat deposits in the body [22]. We demonstrated significantly decreased serum vaspin concentrations in patients with psoriasis compared to the control group. Similar results were also presented by Ataseven et al. in a group of 56 patients with psoriasis [23]. These findings are in contrast to the results presented by Coban et al. who found higher vaspin levels in a group of 35 patients with psoriasis than in controls [24]. Interestingly, Saalbach et al. showed no difference in the serum vaspin concentration between healthy controls and patients with psoriasis [25]. Due to the lack of other studies, observed variances point out the need for further research including more patients followed-up for a longer time. Discussing the role of vaspin in psoriasis we should mention the study of Saalbach et al. who for the first time demonstrated expression of vaspin protein in keratinocytes. Furthermore, they showed that vaspin expression was reduced in lesional psoriatic skin compared with uninvolved skin [25]. They also concluded that vaspin expression by maturing keratinocytes modulates cutaneous immune responses and may be involved in the pathogenesis of psoriasis [26]. Similar results of reduced vaspin levels in psoriatic lesions in relation to healthy skin were also obtained by Sayed et al. [27]. These results highlight the important role of vaspin in psoriasis and the need for further research.

The study showed no correlation between vaspin levels and disease activity determined by PASI. However, we have observed a strong trend in its level in individual
PASI subgroups comparing to the controls. Interestingly, vaspin levels decreased with the severity of the disease. Similar observations of a significantly reduced vaspin concentration in patients with PASI above 10 compared to the group with PASI below 10 were made by Ataseven et al. Researchers suggested that vaspin not only can be used as a marker of the disease but it also may reflect its severity [23]. In the context of above-mentioned reports about decreased vaspin levels in psoriatic lesions, we may hypothesize whether the extent of the involved skin and the severity of lesions may contribute to lowering serum vaspin levels. Similar to our study, the others showed no relationship between the vaspin concentration and the severity of the disease [24, 25]. Thus it seems questionable if vaspin is a reliable marker of intensity of the psoriasis course. This research demonstrated existence of a significant correlation between the vaspin level and BMI of psoriatic patients, no such relations were found in the control group. The lowest median vaspin concentration, significant in relation to healthy volunteers, was noticed in overweight patients and the highest among obese psoriatics however without significance. In contrast, in other reports in patients with psoriasis, no correlation between vaspin and BMI was observed [24, 25]. The data regarding association between vaspin and body weight are inconsistent also in other CMDs. Some researchers have shown that elevated vaspin serum levels are associated with BMI and insulin resistance $[28,29]$. Others associated low vaspin levels with the risk of progression of T2D or have not confirmed the compound between vaspin levels and obesity or metabolic syndrome [30,31]. Due to the inconsistency of data, Feng et al. conducted a meta-analysis in which they revealed significantly increased vaspin levels in T2D patients as well as in obese patients compared to nonobese individuals [32]. These results are to a certain extent reflected in ours, where we presented a significant difference in vaspin levels between BMI groups among patients with psoriasis. It has been suggested that an increase in the vaspin expression may constitute a compensatory mechanism in response to an increase in obesity and insulin resistance [33]. Similarly, we can translate an increased vaspin concentration in overweight or obese patients with psoriasis as observed in our study. Due to a small number of studies assessing vaspin levels in patients with psoriasis we can refer to publications highlighting the presumed involvement of vaspin in the development of CMDs, and thus emphasize the possible association of psoriasis with other chronic inflammatory states as well as indicate the need for further research in this relationship in psoriatics.

As an example, Aust et al. revealed that vaspin serum concentrations were significantly lower in patients with carotid stenosis and they concluded that vaspin could serve as a novel marker of unrecognized symptoms of carotid artery stenosis [34]. Moreover, Li et al. demonstrated 
that a low vaspin serum level predicts coronary artery disease (CAD) and unstable angina pectoris [35]. Above and beyond they suggested a suppressive influence of vaspin on the inflammatory process, which requires further investigations. Based on previous reports and their own studies, Kobat et al. found lower vaspin levels in CAD compared with age-matched subjects who had same symptoms with angiographically normal coronary anatomy and they presumed that low serum vaspin levels may be a predictor of atherosclerotic disease [36]. These outcomes have led us to perform our study and explore the potential diagnostic value of vaspin in psoriasis and perhaps the development of psoriasis comorbidities. Thus, gathering all the data mentioned above along with our preliminary results, vaspin might be a novel protective protein of perhaps predicting CMDs properties. Psoriasis as a systemic disease is characterized by the coexistence of many pathophysiological disorders, including cardiometabolic ones, which may influence the obtained results. Thus, further studies performed on larger groups with stratification according to CAD risk assessment are definitely required.

As we have stated, T2D is usually accompanied by elevated vaspin levels. However, some studies indicate that serum vaspin levels were lower in patients with DM microvascular complications than in patients without them [37]. In relation to these reports, Sathyaseelan et al. conducted an interesting study on a group of patients with diabetes. They showed a reduced concentration of vaspin in patients with acute coronary syndrome (ACS) and hypothesized that decreased serum vaspin acts as a predictor of ACS in patients with T2D [38]. Thus, based on our research, we could assume that a decreased vaspin serum level in patients with psoriasis can contribute to CAD development. Additionally, patients most diseased/most diseased patients may be most predisposed to developing cardiovascular complications. The associations between vaspin and obesity, diabetes or CAD indicate that vaspin may become a potential biomarker of MS.

As vaspin is considered as a marker of MS, more and more studies assessing its concentration in inflammatory diseases have been appearing. One of these diseases is psoriatic arthritis (PsA), common comorbidity in psoriasis, which could share mutual obesity-related complications such as MS, T2D, IR or CVD. Colak et al. determined that vaspin concentration in patients with PsA was higher than in healthy controls, but without any correlation between disease activity and its levels [39]. As demonstrated, various chronic inflammatory diseases may present different vaspin responses reflecting perhaps mutual or compensating nature of the protein.

After 3 months of systemic treatment we observed improvement of skin lesions in all studied patients and attenuation of inflammation expressed in the decrease in inflammatory parameters. We noticed a trend towards the increase in vaspin concentration after treatment.
Noteworthy, vaspin levels remained significantly lower in patients with psoriasis after treatment compared to the control group. Attention should also be paid to the post-therapy increase in vaspin levels in overweight or obese patients, which may indicate the potential benefits of systemic treatment in psoriatics in respect of vaspin levels.

Considering the impact of a particular drug, we found no significant changes in vaspin levels before and after treatment with acitretin or methotrexate. Similarly, Coban et al. did not find changes in vaspin levels before and after MTX treatment of psoriasis patients [24]. These results may indicate a possible lack of effect of the most commonly used systemic medications in psoriasis therapy such as acitretin and methotrexate on the production and metabolism of vaspin or perhaps the existence of other confounding mechanisms. Furthermore, an upward trend in vaspin levels after treatment observed may be due to reduced skin involvement or improvement of the inflammatory state. Referring to the study showing a reduced concentration of vaspin in psoriatic lesions, it was found that improvement of psoriatic plaques by narrowband ultraviolet $B$ radiation is associated with an upregulation of tissue vaspin levels [27].

We demonstrated a significant positive correlation between serum vaspin level and total cholesterol, triglycerides and WBC in psoriatic patients. These findings highlight possible links between vaspin and lipid metabolism as well as its effects on the immune system. Thus vaspin might be another indicator of the pro-atherogenic risk in psoriasis. Interestingly, reported correlations disappear after systemic treatment, what may reflect indirectly its cardioprotective effect. Breitfeld et al. attempted to confirm the association of vaspin with the serum lipid profile in humans.

As presented in other publications regarding vaspin concentration in inflammatory diseases or metabolic disorders, correlations with lipids or inflammatory parameters are ambiguous. Coban et al. in their research based on psoriatic patients found no relations between vaspin and metabolic variables [24]. Aliasghari et al. based on a group of patients with NAFLD noted that vaspin is positively significantly associated with hs-CRP [40]. In contrast, some researches revealed a negative association between hs-CRP and vaspin levels [34, 41]. The numerous, often inconsistent, associations of vaspin with metabolic or inflammatory variables could underline the multidirectional function that it performs in the body.

Regarding omentin, the literature demonstrates that it could play a protective role in obesity-related inflammation [42, 43]. Hence, it arouses a considerable interest of researchers, including our team. We showed an existence of an upward trend in omentin concentration in patients with psoriasis in relation to the controls, however, without any statistical significance. These findings are a bit in agreement with a previous study conducted 
by Coban et al. showing elevated omentin levels in psoriatic patients [24]. Nonetheless, these results are opposite to the majority of studies assessing omentin concentrations in patients with psoriasis, in which a significant decrease in its level was observed [44-48]. The differences may be due to a smaller size of our study group or other confounding factors or methodological nuances. Zhang et al. went a step further and showed not only a decreased plasma omentin concentration in patients with psoriasis but also a reduced expression of this adipokine in the epidermis of psoriatic lesions [46]. Another interesting study was conducted by Xue et al. who showed higher levels of omentin in patients with psoriatic arthritis not only in relation to healthy controls but also to patients with psoriasis without joint involvement. In addition, they found omentin was positively correlated with osteoclast precursors. Therefore, they hypothesized that omentin might contribute to pathogenesis of arthritis [49]. This encourages further studies conducted on patients with psoriasis and PA to assess whether omentin might be a predictor of joint involvement also in psoriasis.

We found no correlation between severity of the disease and the serum omentin level, but we documented a significantly higher level of omentin in patients with severe psoriasis in relation to those with a moderate one. In Coban et al. study, PASI values were significantly positively correlated with serum omentin [24]. Differently from that, in other mentioned studies serum omentin levels negatively correlated with PASI score or similarly as in ours, it showed no dependence between these variables [44-46, 48]. Thus, the relation between omentin and severity of psoriasis remains debatable, however its highest level in most diseased psoriatics might reflect omentin's compensatory effect to be further elucidated.

It was demonstrated that the serum omentin level and omentin gene expressions in visceral adipocytes are decreased in obese cases or in impaired glucose tolerant and T2D subjects compared with healthy controls [50]. Additionally, serum omentin concentration negatively correlated with BMI or waist circumference [50]. In our study, just as Coban et al., we did not find any significant correlations between omentin concentration and BMI of psoriatic patients [24]. Contrarily, Ismail et al. reported that omentin levels inversely correlated with BMI in psoriatics [48]. Similar results were shown by Zhang et al. and Turan et al. [46, 47]. Moreover, a low omentin level had been suggested to be a promising marker of the cardiovascular risk. It was negatively correlated with carotid intima-media thickness and was associated with the prevalence of coronary artery disease [51]. Taking all aforementioned data together, a decreased level of omentin might be considered as a factor which may contribute to development of CMDs in the general population as well as in psoriatic patients. Nevertheless, our study did not confirm previous reports. In addition, we did not observe changes in the concentration of omentin after systemic treatment, also regardless of the drug used. In a study mentioned earlier and performed by Coban et al. the concentration of omentin significantly decreased after 3 months of methotrexate therapy [24]. In turn, Japanese researchers found an increase in omentin levels as a result of treating psoriatic lesions [44]. Definitely further studies are needed in order to explore the potency of omentin and whether it exerts a protective, predicting or compensatory role in the interplay between psoriasis and its comorbidities.

The limitations of our study may result from a relatively small number of patients enrolled in our study and the short duration of follow-up. This may be the reason for the discrepancy of our results compared to previous studies. Undoubtedly, the study points to an important topic for further discussion and subsequent scientific research regarding the role of vaspin and omentin in the development or maintenance of systemic inflammation associated with psoriasis and further development of CMDs.

\section{Conclusions}

In this study, we provide insights into the roles of vaspin and omentin in psoriasis. It is considered that vaspin and omentin play an anti-inflammatory role in the organism but their biological and metabolic functions have not been extensively studied yet. Herein, we demonstrated significantly decreased serum vaspin concentrations and an upward trend in omentin concentration in patients with psoriasis in relation to the healthy subjects. No correlation between vaspin or omentin levels and severity of psoriasis was found. However, we noticed that vaspin levels decreased with the severity of the disease. Reversely, we discovered a higher level of omentin in patients with severe psoriasis in relation to those with a moderate form. Thus omentin might serve as an indicator of metabolically-driven inflammation intensifying within the severity of disease. Research showed a significant correlation between the vaspin level and BMI of psoriatic patients reflecting its role in adiposity and metabolic disorders. A positive relation between vaspin and lipids parameters might reflect its modulating role in CMDs development in psoriatics. Furthermore, its decreased serum level might be linked to metabolic complications in psoriasis. Considering our results as preliminary, the above assumptions need further larger research in order to fully elucidate these relations. After 3 months of systemic treatment with acitretin or methotrexate we noticed a trend towards the increase in vaspin concentration after treatment, however without statistical significance. Omentin levels did not change after therapy. Considering ambiguous literature data, it cannot be clearly stated whether systemic antipsoriatic treatment influences or not the adipokines levels or there 
are some confounding factors, therefore further research is needed. Similarly, various chronic inflammatory diseases present different vaspin and omentin responses. Altogether they come to a conclusion of a multifactorial nature of metabolic disorders and their association with vaspin and omentin. Similar conclusions about importance of vaspin and omentin in the pathogenesis and development of systemic inflammation in psoriatic patients can be made. Summarizing, the importance of vaspin and omentin in psoriasis is not clear and there are a lot of conflicting data requiring in-depth research.

\section{Acknowledgments}

This study was supported by a study grant from the Medical University of Bialystok (Project No. N/ST/ MN/17/002/1149).

\section{Conflict of interest}

The authors declare no conflict of interest.

\section{References}

1. Boenhncke EH, Boehncke S, Tobin Anne-Marie, et al. The 'psoriatic march': a concept of how severe psoriasis may drive cardiovascular comorbidity. Exp Dermatol 2011; 20: 303-7.

2. Abuabara K, Azfar RS, Shin DB, et al. Cause-specific mortality in patients with severe psoriasis: a population-based cohort study in the U.K. Br J Dermatol 2010; 163: 586-92.

3. Hu SC, Lan CE. Psoriasis and Cardiovascular comorbidities: focusing on severe vascular events, cardiovascular risk factors and implications for treatment. Int J Mol Sci 2017; 18: 2211.

4. Hulthe J, Hulten LM, Fagerberg B. Low adipocyte-derived plasma protein adiponectin concentrations are associated with the metabolic syndrome and small dense low-density lipoprotein particles: atherosclerosis and insulin resistance study. Metabolism 2003; 52: 1612-4.

5. Miller IM, Ellervik C, Zarchi K, et al. The association of metabolic syndrome and psoriasis: a population- and hospitalbased cross-sectional study. J Eur Acad Dermatol Venereol 2015; 29: 490-7.

6. Chiricozzi A, Raimondo A, Lembo S, et al. Crosstalk between skin inflammation and adipose tissue-derived products: pathogenic evidence linking psoriasis to increased adiposity. Expert Rev Clin Immunol 2016; 12: 1299-308.

7. Coimbra S, Catarino C, Santos-Silva A. The triad psoriasisobesity adipokine profile. J Eur Acad Dermatol Venereol 2016; 30: 1876-85.

8. Hida K, Wada J, Eguchi J, et al. Visceral adipose tissue-derived serine protease inhibitor: a unique insulin-sensitizing adipocytokine in obesity. Proc Natl Acad Sci USA 2005; 102: 10610-5.

9. Heiker JT, Klöting N, Kovacs P, et al. Vaspin inhibits kallikrein 7 by serpin mechanism. Cell Mol Life Sci 2013; 70: 2569-83.

10. Wada J. Vaspin: a novel serpin with insulin-sensitizing acts. Exp Opin Invest Drugs 2008; 17: 327-33.
11. Yang RZ, Shuldiner AR, Gong DW. Cloning of omentin, a new adipokine from human omental fat tissue. Diabetes 2003; 52: 1-A730.

12. Schäffler A, Neumeier M, Herfarth H, et al. Genomic structure of human omentin, a new adipocytokine expressed in omental adipose tissue. Biochem Biophys Acta 2005; 1732: 96-102.

13. Yang RZ, Lee MJ, Hu H, et al. Identification of omentin as a novel depot-specific adipokine in human adipose tissue: possible role in modulating insulin action. Am J Physiol Endocrinol Metab 2006; 290: 1253-61.

14. Maruyama S, Shibata R, Kikuchi R et al. Fat-derived factor omentin stimulates endothelial cell function and ischemiainduced revascularization via endothelial nitric oxide synthase-dependent mechanism. J Biol Chem 2012; 287: 408-17.

15. Brunetti L, Leone S, Orlando G, et al. Hypotensive effects of omentin-1 related to increased adiponectin and decreased interleukin-6 in intra-thoracic pericardial adipose tissue. Pharmacol Rep 2014; 66: 991-5.

16. Blüher M. Adipose tissue dysfunction in obesity. Exp Clin Endocrinol Diabetes 2009; 117: 241-50.

17. Bays HE. "Sick fat", metabolism disease, and atherosclerosis. Am J Med 2009; 122: S26-S37.

18. Gulcelik NE, Usman A, Gürlek A. Role of adipocytokines in predicting the development of diabetes and its late complications. Endocrine 2009; 36: 397-403.

19. Kralisch S, Blüher M, Paschke R, et al. Adipokines and adipocyte targets in the future management of obesity and the metabolic syndrome. Mini Rev Med Chem 2007; 7: 39-45.

20. Gaal LF, Mertens IL, DeBlock CE. Mechanisms linking obesity with cardiovascular disease. Nature 2006; 444: 875-80.

21. Heron $M$, Hinckley $M$, Hoffman $M$, et al. Impact of obesity and smoking on psoriasis presentation and management. Arch Dermatol 2005; 141: 1527-34.

22. Kloting N, Berndt J, Kralisch S, et al. Vaspin gene expression in human adipose tissue: association with obesity and type 2 diabetes. Biochem Biophys Res Commun 2006; 339: 430-6.

23. Ataseven A, Kesli R. Novel inflammatory markers in psoriasis vulgaris: vaspin, vascular adhesion protein-1 (VAP-1), and YKL-40. G Ital Dermatol Venereol 2016; 151: 244-50.

24. Coban M, Tasli L, Turgut S, et al. Association of adipokines, insulin resistance, hypertension and dyslipidemia in patients with psoriasis vulgaris. Ann Dermatol 2016; 28: 74-9.

25. Saalbach A, Vester K, Rall K, et al. Vaspin - a link of obesity and psoriasis? Exp Dermatol 2012; 21: 299-319.

26. Saalbach A, Tremel J, Herbert D, et al. Anti- inflammatory action of keratinocyte-derived vaspin. Relevance for the pathogenesis of psoriasis. Am J Pathol 2016; 186: 639-51.

27. Sayed KS, Said ER, Mohammed FN, et al. Down-regulation of tissue levels of serine protease inhibitor (vaspin) in psoriasis vulgaris patients: a possible mechanism of narrowband ultraviolet B radiation. Arch Dermatol Res 2019; 311: 637-41.

28. Teshigawara S, Wada J, Hida K, et al. Serum vaspin concentrations are closely related to insulin resistance, and rs77060950 at SERPINA12 genetically defines distinct group with higher serum levels in Japanese population. J Clin Endocrinol Metab 2012; 97: E1202-E1207.

29. Youn BS, Kloting N, Kratzsch J, et al. Serum vaspin concentrations in human obesity and type 2 diabetes. Diabetes 2008; 57: 372-7.

30. Jian W, Peng W, Xiao S, et al. Role of serum vaspin in progression of type 2 diabetes: a 2-year cohort study. PLoS One 2014; 9: e94763. 
31. Kim JM, Kim TN, Won JC. Association between serum vaspin level and metabolic syndrome in healthy Korean subjects. Metab Syndr Relat Disord 2013; 11: 385-91.

32. Feng R, Li Y, Wang C, et al. Higher vaspin levels in subjects with obesity and type 2 diabetes mellitus: a meta-analysis. Diabetes Res Clin Pract 2014; 106: 88-94.

33. Klöting N, Kovacs P, Kern M, et al. Central vaspin administration acutely reduces food intake and has sustained blood glucose-lowering effects. Diabetologia 2011; 54: 1819-23.

34. Aust G, Richter O, Rohm S, et al. Vaspin serum concentration in patients with carotid stenosis. Atherosclerosis 2009; 204: 262-6.

35. Li HL, Peng WH, Ciu ST, et al. Vaspin plasma concentrations and mRNA expressions in patients with stable and unstable angina pectoris. Clin Chem Lab Med 2011; 49: 1547-54.

36. Kobat MA, Celik A, Balin M, et al. The investigation of serum vaspin level in atherosclerothic coronary artery disease. J Clin Med Res 2012; 4: 110-3.

37. Gulcelik NE, Karakaya J, Gedik A, et al. Serum vaspin levels in type 2 diabetic women in relation to microvascular complications. Eur J Endocrinol Eur Fed Endocr Soc 2009; 160: 65-70.

38. Sathyaseelan AJ, Adole PS, Wyawahare M, et al. Assesment of serum VASPIN levels among type 2 diabetes mellitus patients with or without acute coronary syndrome. J Clin Diagn Res 2016; 10: BC07-10.

39. Colak S, Omma A, Sandikci SC, et al. Vaspin, neutrophil gelatinase-associated lipocalin and apolipoprotein levels in patients with psoriatic arthritis. Bratisl Lek Listy 2019; 120: 65-9.

40. Aliasghari F, Izadi A, Jabbari M, et al. Are vaspin and omentin-1 related to insulin resistance, blood pressure and inflammation in NAFLD patients? J Med Biochem 2018; 37: 470-5.

41. Genc H, Dogru T, Tapan S, et al. Circulating vaspin and its relationship with insulin sensitivity, adiponectin, and liver histology in subjects with non-alcoholic steatohepatitis. Scand J Gastroenterol 2011; 46: 1355-61.

42. Cătoi AF, Suciu S, Pârvu AE et al. Increased chemerin and decreased omentin-1 levels in morbidly obese patients are correlated with insulin resistance, oxidative stress and chronic inflammation. Clujul Med 2014; 87: 19-26.

43. Zabetian-Targhi F, Mirzaei K, Keshavarz SA, et al. Modulatory role of omentin-1 in inflammation: cytokines and dietary intake. J Am Coll Nutr 2016; 35: 670-8.

44. Takahashi H, Tsuji H, Honma M, et al. Increased plasma resistin and decreased omentin levels in Japanese patients with psoriasis. Arch Dermatol Res 2013; 305: 113-6.

45. Bakry OA, Farargy SE, Ghanayem N, et al. Serum omentin-1 in psoriasis. Indian J Dermatol 2018; 63: 85-7.

46. Zhang C, Zhu KJ, Liu JL, et al. Omentin-1 plasma levels and omentin-1 expression are decreased in psoriatic lesions of psoriasis patients. Arch Dermatol Res 2015; 307: 455-9.

47. Turan H, Yaykasli KO, Soguktas H, et al. Omentin serum levels and omentin gene Val109Asp polymorphism in patients with psoriasis. Int J Dermatol 2014; 53: 601-5.

48. Ismail SA, Mohamed SA. Serum levels of visfatin and omentin-1 in patients with psoriasis and their relation to disease severity. Br J Dermatol 2012; 167: 436-9.

49. Xue Y, Jiang L, Cheng Q, et al. Adipokines in psoriatic arthritis patients: the correlations with osteoclast precursors and bone erosions. PLoS One 2012; 7: e46740.

50. De Souza Batista CM, Yang RZ, Lee MJ, et al. Omentin plasma levels and gene expression are decreased in obesity. Diabetes 2007; 56: 1655-61.
51. Shibata R, Ouchi N, Takahashi R, et al. Omentin as a novel biomarker of metabolic risk factors. Diabetol Metab Syndr 2012; 4: 37. 